\title{
Multi-walled carbon nanotubes-induced alterations in microRNA let-7 and its targets activate a protection mechanism by conferring a developmental timing control
}

\author{
Li Zhao ${ }^{1}$, Hanxiao Wan', Qizhan Liu² and Dayong Wang ${ }^{1 *}$ (D)
}

\begin{abstract}
Background: Multi-walled carbon nanotubes (MWCNTs) have been produced and applied for diverse purposes. MWCNTs can potentially cause the adverse effects on organisms. MicroRNA let-7 and its targets of HBL-1 and LIN-41 play a central role in regulating developmental timing by acting as a developmental switch. The sequence of let-7 and the underlying mechanisms for let-7 in the control of developmental timing are conserved among different species. In this study, we investigated the potential effect of MWCNTs exposure on the molecular basis for developmental timing mediated by let-7 and its targets of HBL-1 and LIN-41.
\end{abstract}

Results: MWCNTs exposure decreased let-7 expression, and increased expressions of hbl-1 and lin-41. let-7 mutant was resistant to MWCNTs toxicity, whereas hbl-1 or lin-41 mutant was susceptible to MWCNTs toxicity. hbl-1 or lin-41 mutant suppressed the resistance of let-7 mutant to MWCNTs toxicity. HBL-1 acted upstream of TIR-1, an adaptor protein, and SYM-1, a protein containing leucine-rich repeats, and ALG-1 and ALG-2, two RDE-1 proteins related to RNA interference (RNAi) pathway, acted upstream of LIN-41 to regulate MWCNTs toxicity. Moreover, we identified a feedback loop between let-7 and its targets of HBL-1 and LIN-41 in the regulation of MWCNTs toxicity. The observed increase in let-7::GFP expression in MWCNTs exposed nematodes with mutation of hbl-1 or lin-41 implied that a feedback mechanism may exist to strengthen the function of let-7 suppression in protecting the animals from MWCNTs toxicity.

Conclusions: Our results demonstrate the protection function of let-7 suppression for animals from MWCNTs toxicity by conferring a robust developmental timing control. Our results highlight the importance of molecular basis for developmental timing in influencing MWCNTs toxicity.

Keywords: let-7, Multi-walled carbon nanotubes, Developmental timing, Nanotoxicity, Caenorhabditis elegans

\section{Background}

Multi-walled carbon nanotubes (MWCNTs), one member of the carbon-based engineered nanomaterials (ENMs), have numerous unique physicochemical properties, such as high electrical conductivity and thermal conductivity [1]. MWCNTs have been produced in bulk for diverse purposes, and can be potentially used in several fields, including drug delivery, heterogeneous

\footnotetext{
* Correspondence: dayongw@seu.edu.cn

${ }^{1}$ Key Laboratory of Environmental Medicine Engineering in Ministry of Education, Medical School, Southeast University, Nanjing 210009, China

Full list of author information is available at the end of the article
}

catalysis to optoelectronic device development, and environmental remediation [2-4]. With the increase in MWCNTs manufacture, the exposure possibility of human and environmental organisms to MWCNTs is also increased $[5,6]$. A series of in vitro and in vivo studies have demonstrated several aspects of MWCNTs toxicity on organisms, such as the pulmonary toxicity, through induction of oxidative stress and/or inflammation $[7,8]$.

Caenorhabditis elegans is a classic non-mammalian model animal [9]. Meanwhile, largely due to its sensitivity to environmental toxicants [10-12], C. elegans has already been widely used for toxicity assessment 
and toxicological study of various environmental toxicants, including carbon-based ENMs [13-17]. In nematodes, MWCNTs exposure could cause the damage on the functions of both primary targeted organs (such as intestine) and secondary targeted organs (such as neurons and reproductive organs) [18-21]. After exposure, MWCNTs could be potentially translocated into the secondary targeted organs, such as reproductive organs, through the intestinal barrier in nematodes [18, 21-23].

microRNAs (miRNAs) are a class of short noncoding RNAs, and can exhibit their biological functions by inhibiting the expression of targeted genes posttranscriptionally [24]. In nematodes, miRNAs have been shown to play important functions in regulating the toxicity of certain carbon-based ENMs, such as MWCNTs and graphene oxide (GO) [25-29]. For example, mir-355 may regulate the MWCNTs toxicity by inhibiting the function of its target of DAF-2, an insulin receptor [28]. The activated mir-259 may protect the nematodes from MWCNTs toxicity by inhibiting the function of its target of RSKS-1, a putative ribosomal protein S6 kinase [29]. In C. elegans, let-7 is one of the founding members of the miRNA family firstly identified via forward genetic screen [30]. It has been shown that let- 7 regulates the timing of larval and adult transition by acting as a developmental switch, and the sequence and functions of let-7 are conserved among different species [30,31]. During the control of transition of developmental timing, let-7 suppresses the expression and function of its direct targets of HBL- 1 and LIN-41 $[30,31]$. In this study, we investigated the potential effect of MWCNTs exposure on the molecular basis for developmental timing mediated by let-7 and its targets of HBL-1 and LIN-41 using in vivo assay system of $C$. elegans. Our results demonstrated that the miRNA let-7 could be decreased by MWCNTs exposure, and was required for the regulation of MWCNTs toxicity. let-7 further regulated
MWCNTs toxicity by suppressing the functions of HBL-1 signaling and LIN-41 signaling. Our data imply that the let-7-mediated molecular basis for the control of developmental-timing transition is dysregulated by MWCNTs exposure, and this molecular basis may be required for the control of MWCNTs toxicity in organisms.

\section{Results}

\section{Effect of MWCNTs exposure on let-7 expression}

Our previous studies have demonstrated that prolonged exposure (from L1-larvae to young adults) to MWCNTs at concentrations more than $1 \mu \mathrm{g} / \mathrm{L}$ could result in the significant induction of intestinal ROS production and decrease in locomotion behavior in nematodes $[18,21]$. The concentration of $10 \mu \mathrm{g} / \mathrm{L}$ was selected as the working concentration for MWCNTs in this study. In C. elegans, let-7 is expressed in almost all tissues except the germiline [32]. Using the transgenic strain of $z a E \times 5[$ let-7::GFP], we observed that prolonged exposure to MWCNTs $(10 \mu \mathrm{g} / \mathrm{L})$ significantly decreased the let-7::GFP expression in the body of nematodes (Fig. 1a). We further focused on the intestinal cells to compare the relative fluorescence intensity of let-7::GFP between control and MWCNTs exposed nematodes. After MWCNTs $(10 \mu \mathrm{g} / \mathrm{L})$ exposure, the intestinal let-7::GFP was significantly decreased compared with control (Fig. 1b).

Mutation of let-7 induced a resistance to MWCNTs toxicity Using the loss-of-function mutant of let-7(mg279), we next investigated the effect of let-7 mutation on the MWCNTs toxicity. Under normal conditions, loss-offunction mutation of let-7 does not induce significant induction of intestinal reactive oxygen species (ROS) production and alteration in locomotion behavior (Fig. 2). After MWCNTs exposure, we found that the loss-of-function mutant of let-7(mg279) was resistant to MWCNTs toxicity in inducing intestinal ROS
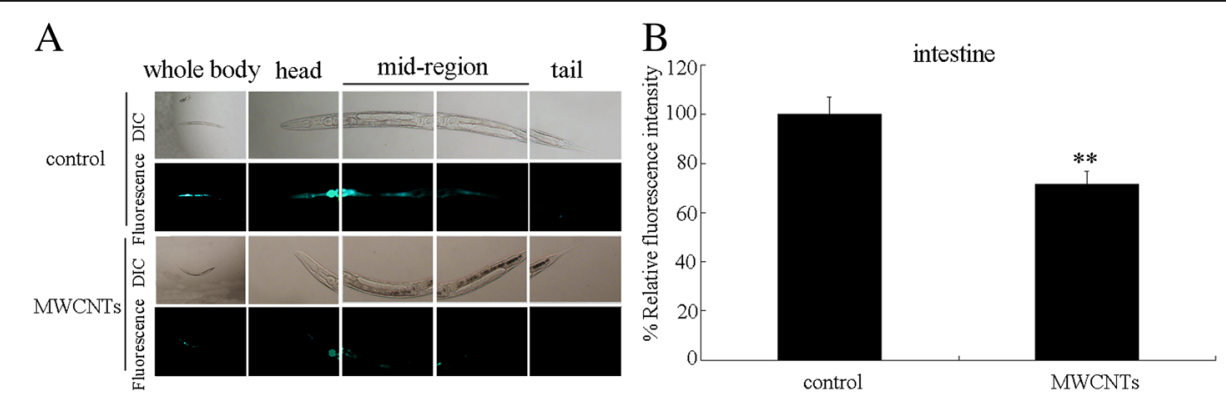

Fig. 1 Effect of MWCNTs exposure on let-7::GFP expression. a Comparison of let-7::GFP expression in control and MWCNTs exposed nematodes. b Comparison of intestinal let-7::GFP expression in control and MWCNTs exposed nematodes. Thirty nematodes were examined per treatment. Prolonged exposure was performed from L1-larvae to young adults. Exposure concentration of MWCNTs was $10 \mu \mathrm{g} / \mathrm{L}$. Bars represent means \pm SD. ${ }^{* *} P<0.01$ vs control 

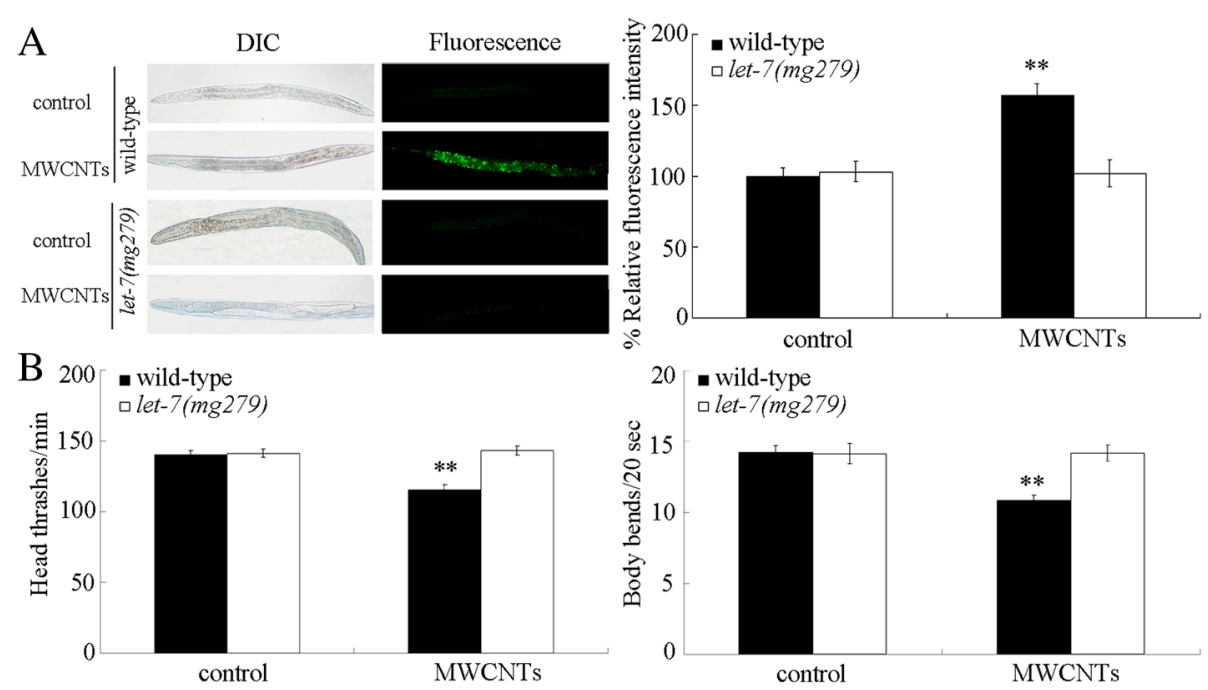

Fig. 2 Effects of let-7 mutation on MWCNTs toxicity. a Effects of let-7 mutation on MWCNTs toxicity in inducing intestinal ROS production. Sixty nematodes were examined per treatment. $\mathbf{b}$ Effects of let-7 mutation on MWCNTs toxicity in decreasing locomotion behavior. Sixty nematodes were examined per treatment. Prolonged exposure was performed from L1-larvae to young adults. Exposure concentration of MWCNTs was $10 \mu \mathrm{g} / \mathrm{L}$. Bars represent means $\pm \mathrm{SD} .{ }^{* *} P<0.01$ vs control

production and in decreasing locomotion behavior (Fig. 2). Therefore, mutation of let-7 may induce a resistance to MWCNTs toxicity.

let-7 mutation increased the expressions of its targets of HBL-1 and LIN-41 in MWCNTs exposed nematodes

During the development, HBL-1 and LIN-41 have been identified as the direct targets for let-7 in the control of developmental-timing transition [30, 31]. Under normal conditions, let-7 mutation increases the expression of its targets of HBL-1 and LIN-41 [30, 31]. Moreover, let-7 mutation significantly increased the expressions of $h b l-1$ and lin-41 in MWCNTs exposed nematodes (Additional file 1: Figure S1A). Additionally, MWCNTs could significantly increase the expressions of $h b l-1$ and $l i n-41$ (Additional file 1: Figure S1B).

\section{Mutation of $h b l-1$ or lin-41 induced a susceptibility to MWCNTs toxicity}

We further investigated the effect of $h b l-1$ or lin-41 mutation on MWCNTs toxicity. Under normal conditions, mutation of $h b l-1$ or lin-41 does not result in significant induction of intestinal ROS production and decrease in locomotion behavior (Fig. 3). Using intestinal ROS production and locomotion behavior as the endpoints, we found that mutation of hbl-1 or lin-41 caused the more severe MWCNTs toxicity in inducing intestinal ROS production and in decreasing locomotion behavior compared with those in MWCNTs exposed wild-type nematodes (Fig. 3). Therefore, mutation of $h b l-1$ or lin-41 may induce a susceptibility to MWCNTs toxicity.
Genetic interaction between let-7 and $h b l-1$ or lin-41 in the regulation of MWCNTs toxicity

To further confirm the role of let-7 and its targets in regulating MWCNTs toxicity, we next investigated the genetic interaction between let-7 and $h b l-1$ or $l i n-41$ in the regulation of MWCNTs toxicity. Under normal conditions, the double mutants of $h b l-1(m g 285) l e t-7(m g 279)$ and lin-41(ma104);let-7(mg279) did not show a significant induction of intestinal ROS production and an obvious alteration in locomotion behavior (Fig. 4). After MWCNTs exposure, mutation of $h b l-1$ or $l i n-41$ could effectively cause the significant induction of intestinal ROS production and decrease in locomotion behavior in let-7(mg279) mutant nematodes (Fig. 4). That is, mutation of $h b l-1$ or lin-41 could effectively suppress the resistance of let-7(mg279) mutant to MWCNTs toxicity in inducing intestinal ROS production and in decreasing locomotion behavior.

\section{Identification of downstream targets for HBL-1 in the regulation of MWCNTs toxicity}

In C. elegans, totally 13 genes (T01D3.6, F13B12.4, ugt18, cpt-4, clec-60, F28H1.1, W04G3.3, K12B6.3, nurf-1, sym-1, tir-1, $n h x-3$, and zig-4) were significantly increased (more than 2.5 fold changes) by $h b l-1$ overexpression under normal conditions [33]. After MWCNTs expression, the expressions of tir-1, sym-1, and $l p r-4$ were significantly decreased by $h b l-1$ mutation (Fig. 5a). In C. elegans, tir-1 encodes a Toll-interleukin 1 receptor (TIR) domain adaptor protein, sym-1 encodes a protein containing 15 contiguous leucine-rich repeats (LRRs), and $l p r-4$ encodes a Lipocalin-related protein. 

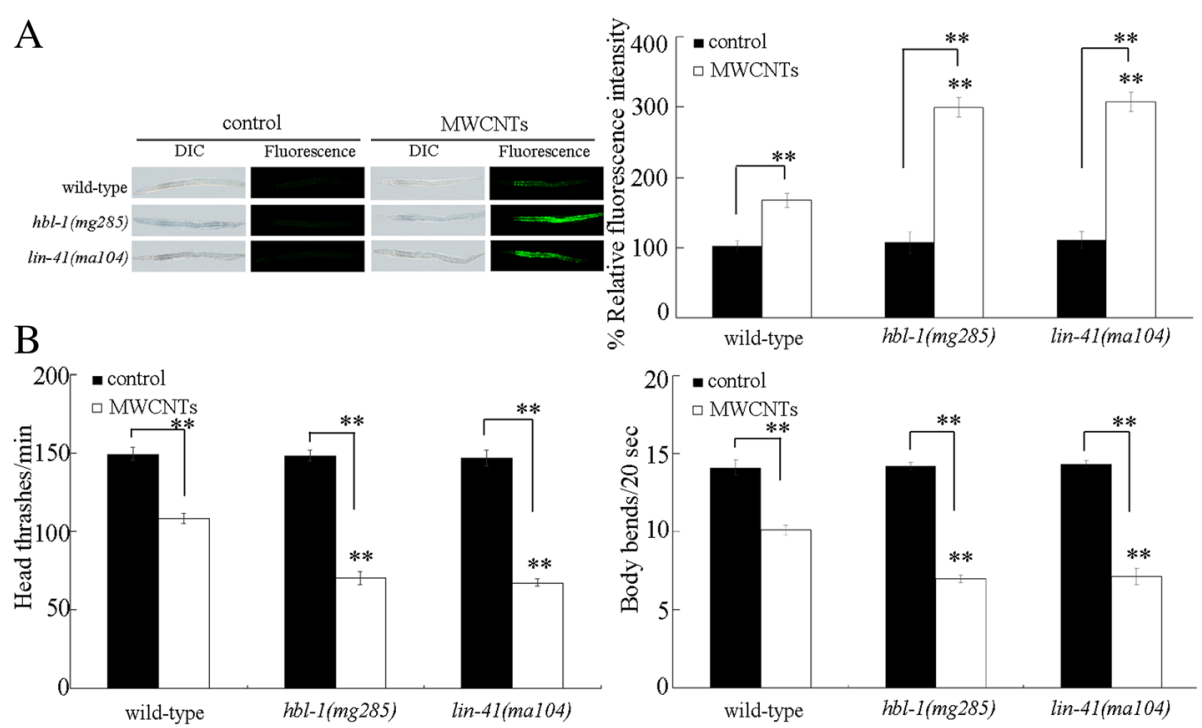

Fig. 3 Effects of hbl-1 or lin-41 mutation on MWCNTs toxicity. a Effects of hbl-1 or lin-41 mutation on MWCNTs toxicity in inducing intestinal ROS production. Sixty nematodes were examined per treatment. $\mathbf{b}$ Effects of $h b l-1$ or lin-41 mutation on MWCNTs toxicity in decreasing locomotion behavior. Sixty nematodes were examined per treatment. Prolonged exposure was performed from L1-larvae to young adults. Exposure concentration of MWCNTs was $10 \mu \mathrm{g} / \mathrm{L}$. Bars represent means $\pm \mathrm{SD} .{ }^{* *} \mathrm{P}<0.01 \mathrm{vs}$ wild-type (if not specially indicated)

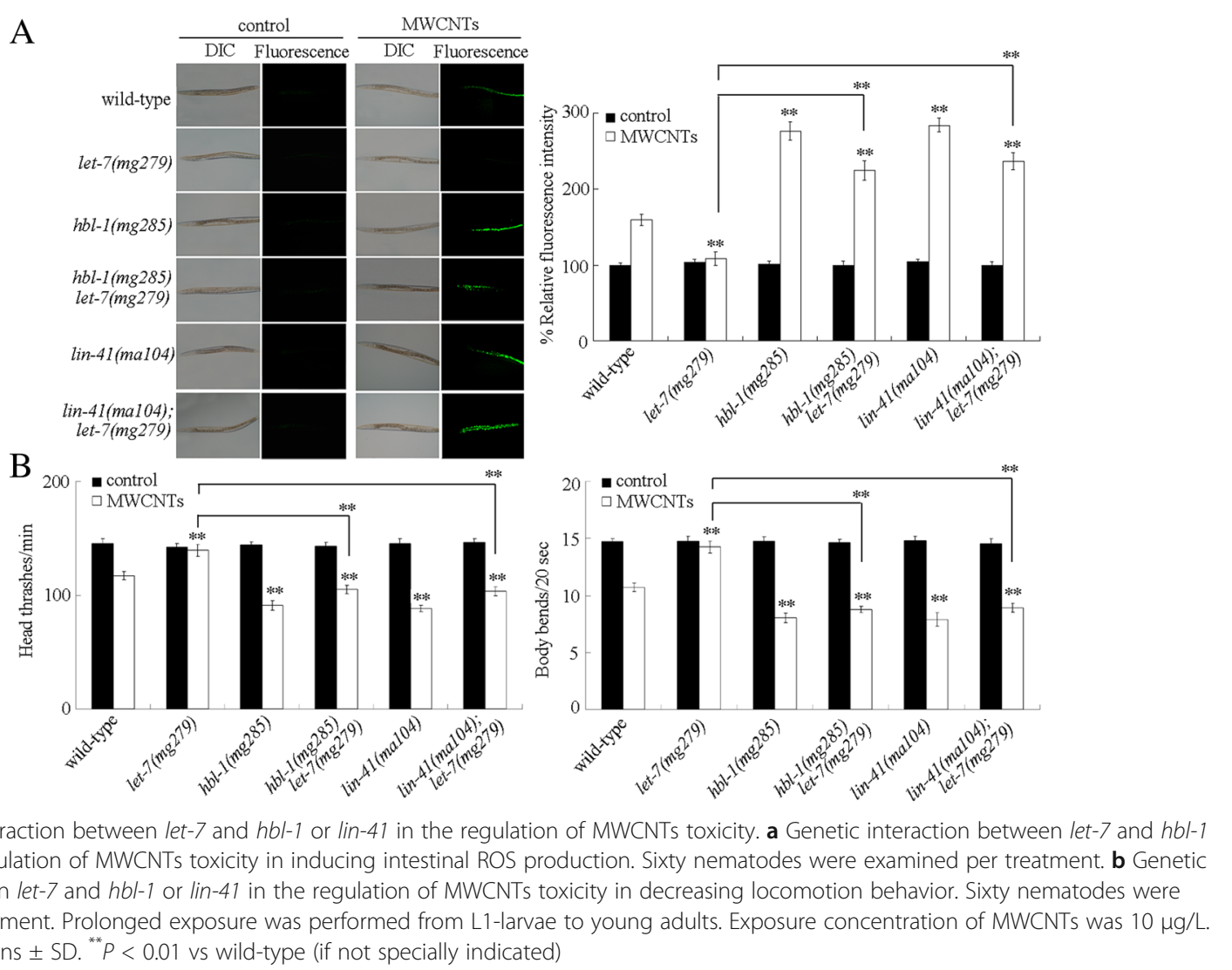




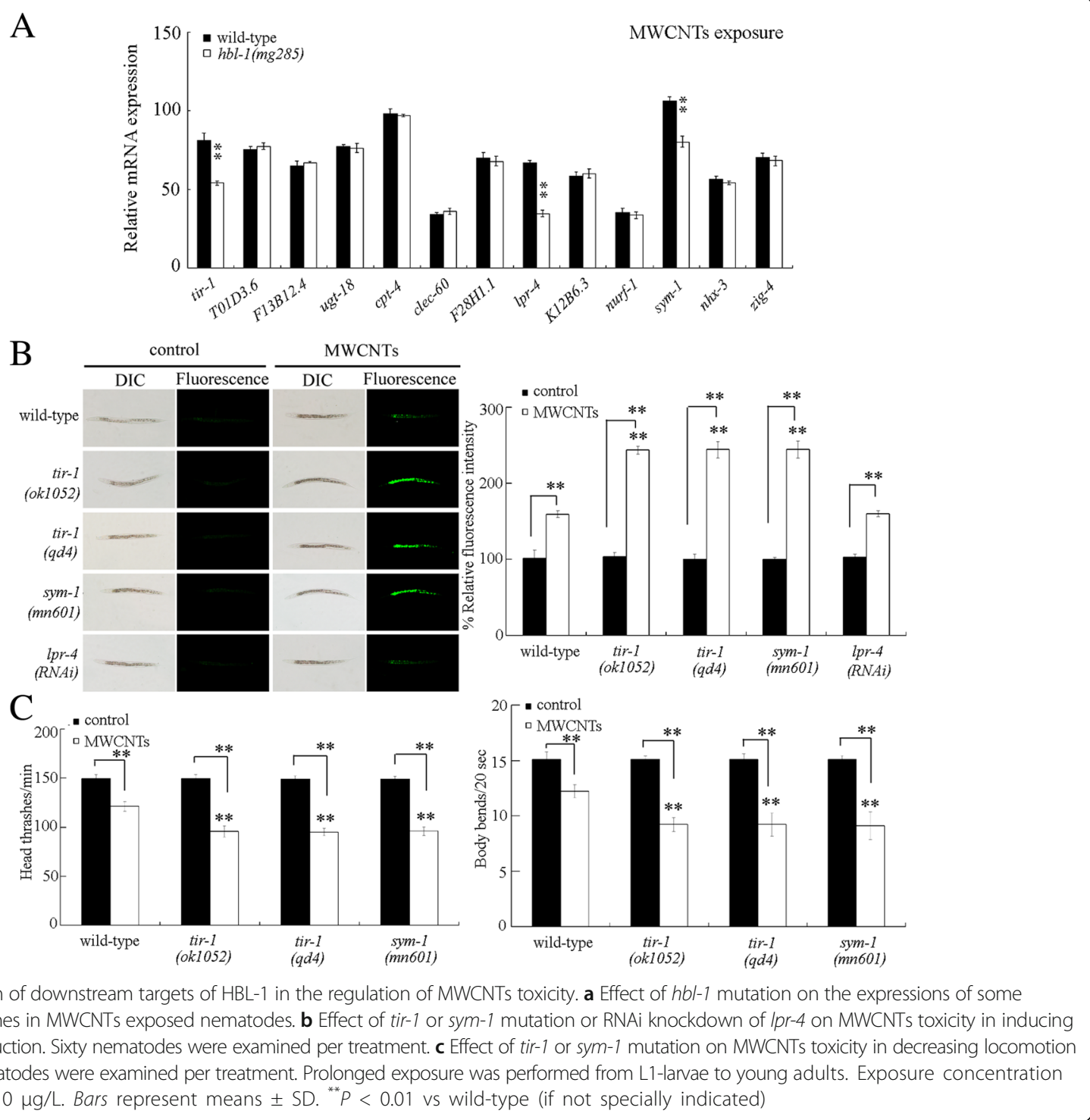

In MWCNTs exposed nematodes, mutation of tir-1 or sym-1 resulted in the more severe induction of intestinal ROS production compared with that in MWCNTs exposed wild-type nematodes (Fig. 5b). In contrast, mutation of $l p r-4$ did not significantly affect the MWCNTs toxicity in inducing intestinal ROS production (Fig. 5b). Moreover, mutation of tir-1 or sym-1 led to the more severe decrease in locomotion behavior in MWCNTs exposed nematodes compared with that in MWCNTs exposed wild-type nematodes (Fig. 5c). These results suggest that mutation of tir-1 or sym-1 may induce a susceptibility to MWCNTs toxicity.

To further investigate the genetic interaction between HBL-1 and TIR-1 or SYM-1 in the regulation of MWCNTs toxicity, we generated the transgenic strain of Ex(Phbl-1-hbl-1) overexpressing HBL-1 in nematodes. The transgenic strain of Ex(Phbl-1-hbl-1) was resistant to MWCNTs toxicity in inducing intestinal ROS production and in decreasing locomotion behavior (Fig. 6). Moreover, we found that mutation of tir-1 or sym-1 significantly increased the induction of intestinal ROS production and decreased the locomotion behavior in MWCNTs exposed transgenic strain of Ex(Phbl-1-hbl-1) (Fig. 6). Therefore, mutation of tir-1 or sym-1 may suppress the resistance of transgenic strain of $E x($ Phbl-1-hbl-1) to MWCNTs toxicity.

Genetic interaction between LIN-41 and ALG-1 or ALG-2 in the regulation of MWCNTs toxicity

In C. elegans, alg- 1 and alg-2 encode two members of RDE-1 proteins, and lin-41 mutations could inhibit the retarded heterochronic phenotypes caused by alg-1/alg-2 RNA interference (RNAi) [34]. We further examined the genetic interaction between 

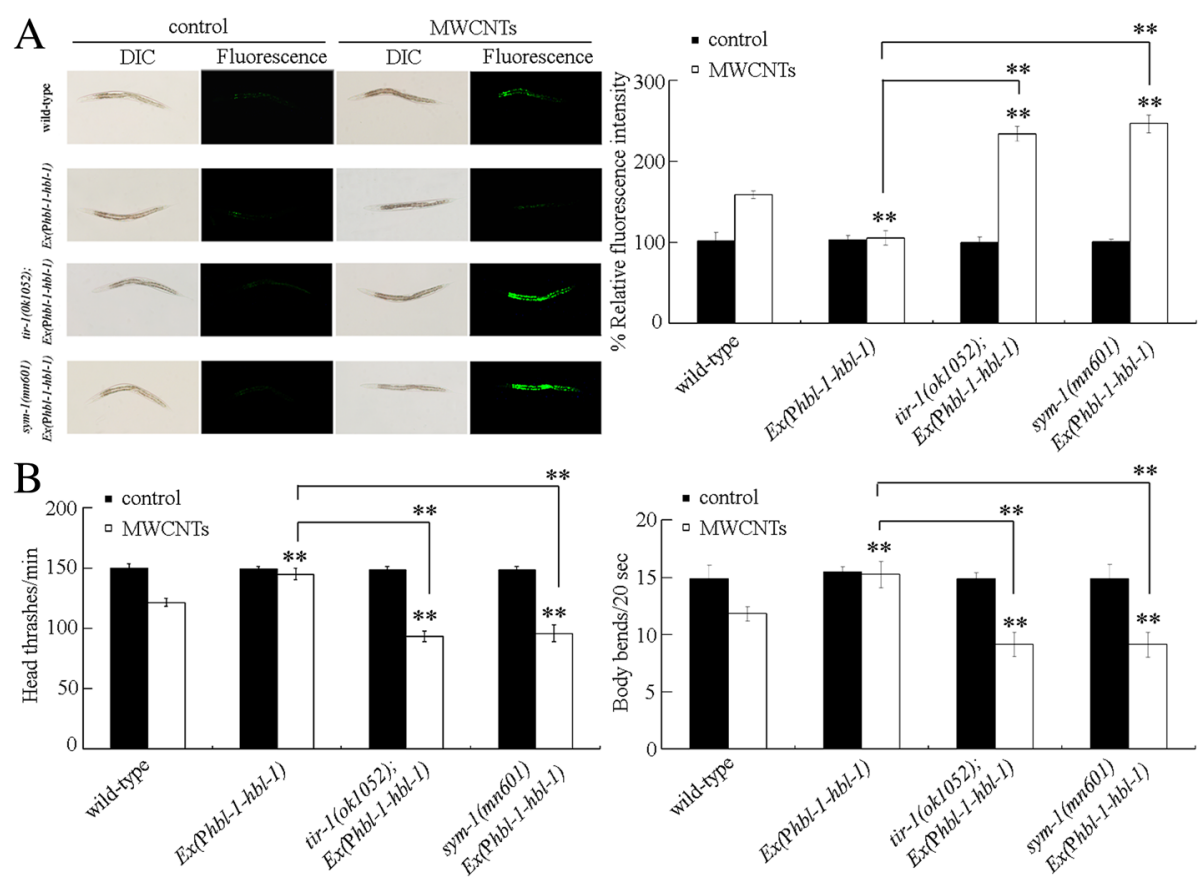

Fig. 6 Genetic interaction between HBL-1 and TIR-1 or SYM-1 in the regulation of MWCNTs toxicity. a Genetic interaction between HBL-1 and TIR-1 or SYM-1 in the regulation of MWCNTs toxicity in inducing intestinal ROS production. Sixty nematodes were examined per treatment. $\mathbf{b}$ Genetic interaction between HBL-1 and TIR-1 or SYM-1 in the regulation of MWCNTs toxicity in decreasing locomotion behavior. Sixty nematodes were examined per treatment. WT, wild-type. Prolonged exposure was performed from L1-larvae to young adults. Exposure concentration of MWCNTs was 10 Mg/L. Bars represent means $\pm \mathrm{SD} .{ }^{* *} P<0.01$ vs wild-type (if not specially indicated)

LIN-41 and ALG-1 or ALG-2 in the regulation of MWCNTs toxicity. After MWCNTs exposure, we found that mutation of alg-1 or alg-2 could induce a resistance to MWCNTs toxicity in inducing intestinal ROS production and in decreasing locomotion behavior (Fig. 7). Moreover, after MWCNTs exposure, lin-41 mutation could significantly suppress the resistance of $\operatorname{alg-1}(g k 214)$ or alg-2(ok304) mutant to MWCNTs toxicity in inducing intestinal ROS production and in decreasing locomotion behavior (Fig. 7). These results suggest that ALG-1 or ALG-2 may act upstream of LIN-41 in the regulation of MWCNTs toxicity.

\section{A feedback loop between let-7 and HBL-1 or LIN-41 in the regulation of MWCNTs toxicity}

It was reported that the let-7 transcription can be temporally regulated by one of its targets, $h b l-1$ [35]. We further found that, besides the $h b l-1$, mutation of lin-41 also significantly increased the expression of let-7::GFP (Fig. 7). Mutation of $h b l-1$ could cause the more significant increase in the expression of let-7::GFP compared with that in nematodes with mutation of lin-41 (Fig. 8). Moreover, mutation of $h b l-1$ or lin- 41 also significantly increased the expression of let-7::GFP in MWCNTs exposed nematodes (Fig. 8).

\section{Discussion}

In this study, we examined the potential effect of MWCNTs exposure on the molecular basis for developmental timing using the in vivo assay system of $C$. elegans. In $C$. elegans, the miRNA let-7 and its direct targets of HBL-1 and LIN-41 regulate the transition of developmental timing [30, 31]. After MWCNTs exposure, we observed the decreased expression of let$7:: G F P$ (Fig. 1a). Moreover, we detected the increased expressions of $h b l-1$ and $l i n-41$ in MWCNTs exposed nematodes (Additional file 1: Figure S1B). Therefore, MWCNTs may affect the molecular basis for developmental timing in animals.

Meanwhile, we investigated the potential role of let-7 in the regulation of MWCNTs toxicity. We found that loss-of-function of let-7 resulted in the formation of resistance to MWCNTs toxicity in inducing intestinal ROS production and in decreasing locomotion behavior (Fig. 2). Therefore, after MWCNTs exposure, the decreased let-7 may mediate a protection mechanism for nematodes against the MWCNTs toxicity. More recently, it was also reported that Pseudomonas aeruginosa infection could suppress the let-7 expression, and loss-of-function mutation of let-7 enhanced the innate immune response of nematodes to $P$. aeruginosa infection [36, 37]. The induced decrease in let-7 expression may be helpful for nematodes against the adverse 

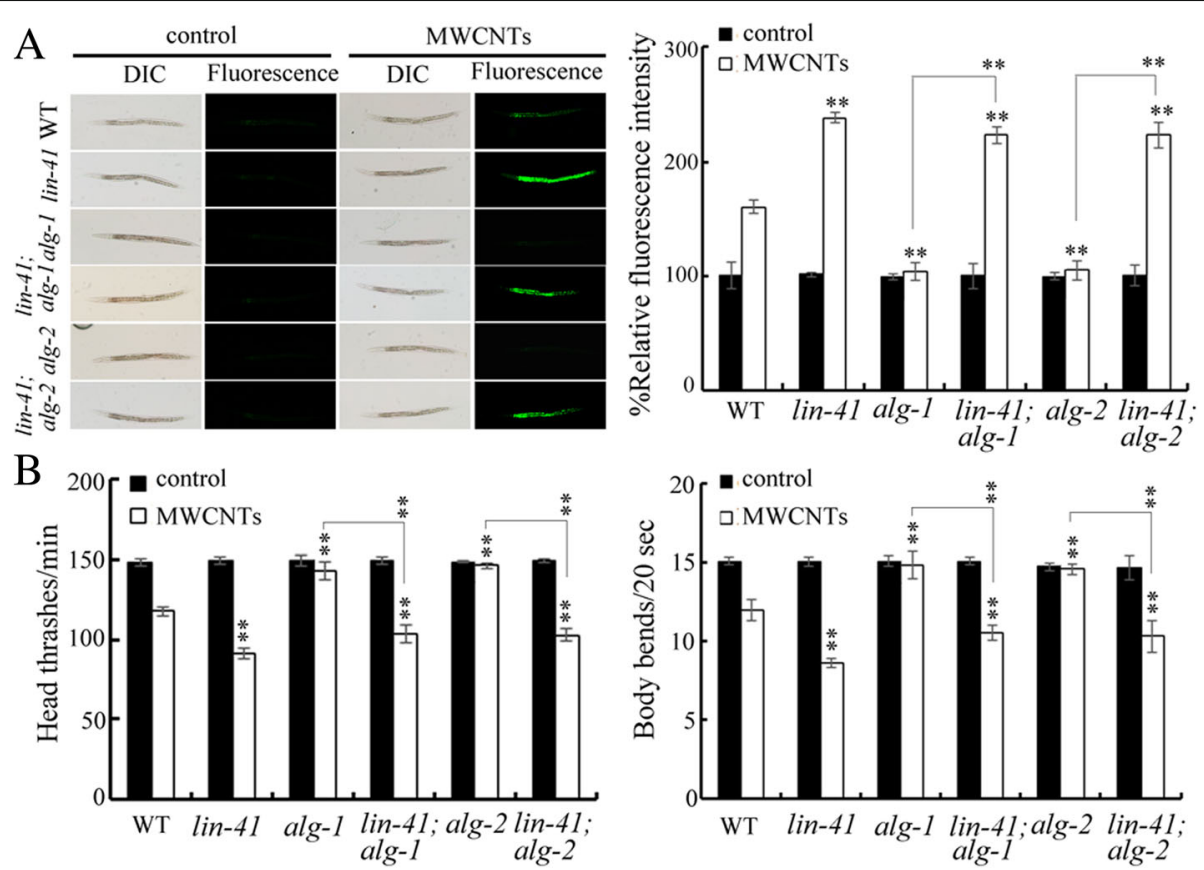

Fig. 7 Genetic interaction between LIN-41 and ALG-1 or ALG-2 in the regulation of MWCNTs toxicity. a Genetic interaction between LIN-41 and ALG-1 or ALG-2 in the regulation of MWCNTs toxicity in inducing intestinal ROS production. Sixty nematodes were examined per treatment. $\mathbf{b}$ Genetic interaction between LIN-41 and ALG-1 or ALG-2 in the regulation of MWCNTs toxicity in decreasing locomotion behavior. Sixty nematodes were examined per treatment. WT, wild-type. Prolonged exposure was performed from L1-larvae to young adults. Exposure concentration of MWCNTs was $10 \mu \mathrm{g} / \mathrm{L}$. Bars represent means $\pm S D .{ }^{* *} P<0.01$ vs wild-type (if not specially indicated)

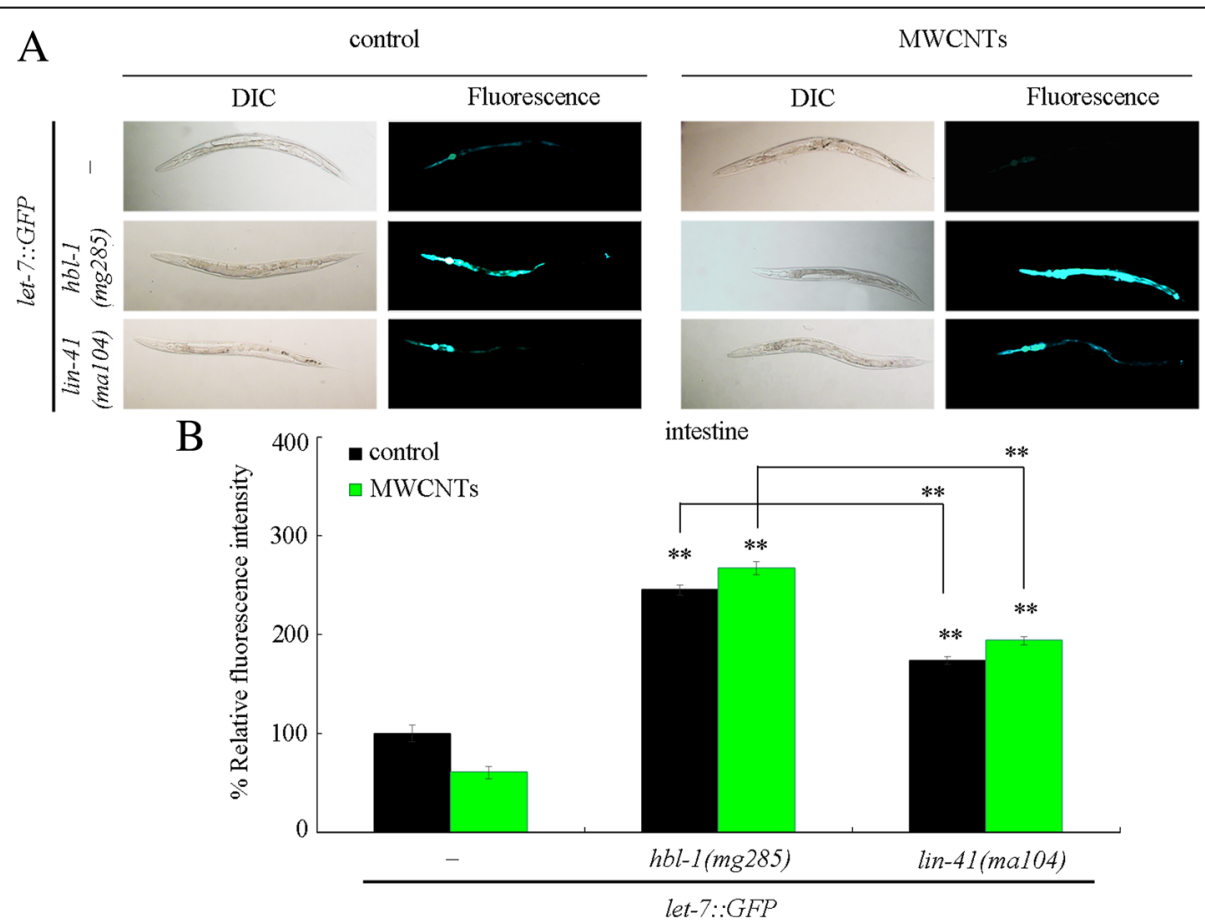

Fig. 8 Effect of mutation of hbl-1 or lin-41 on let-7::GFP expression in MWCNTs exposed nematodes. a Comparison of let-7::GFP expression. b Comparison of intestinal let-7:GFP expression. "-", nematodes without mutation of $h b l-1$ or lin-41. Thirty nematodes were examined per treatment. Prolonged exposure was performed from L1-larvae to young adults. Exposure concentration of MWCNTs was $10 \mu \mathrm{g} / \mathrm{L}$. Bars represent means \pm SD. ${ }^{* *} P<0.01$ vs nematodes without mutation of $\mathrm{hbl}-1$ or lin- 41 (if not specially indicated) 
effects from exposure to environmental toxicants or environmental pathogens.

For the normally identified targets of let-7, HBL-1 and LIN-41 [30, 31], we found that mutation of hbl-1 or lin41 caused the formation of a susceptibility to MWCNTs in inducing intestinal ROS production and in decreasing locomotion behavior (Fig. 3). In C. elegans, hbl-1 encodes a $\mathrm{C} 2 \mathrm{H} 2$-type zinc finger transcriptional factor, and lin-41 encodes a Ring finger-B box-Coiled coil (RBCC) protein. Both HBL-1 and LIN-41 are heterochronic proteins that play an essential role in the regulation of developmental timing during the postembryonic development $[31,38]$. Our results imply that let-7 and its targets may affect the MWCNTs toxicity by conferring a robust developmental timing control.

In C. elegans, $h b l-1$ or lin- 41 translation is negatively regulated by the let-7 that binds to the site(s) in the $h b l$ 1 3'UTR or lin-41 3'UTR [30, 31, 38]. In this study, we further observed that both the $h b l-1$ and the $l i n-41$ were increased by let-7 mutation in MWCNTs exposed nematodes (Additional file 1: Figure S1A). Moreover, the resistance of let-7(mg279) mutant to MWCNTs toxicity in inducing intestinal ROS production and in decreasing locomotion behavior could be suppressed by $h b l-1 \mathrm{mu}-$ tation or lin-41 mutation (Fig. 4). Similarly, the resistance of let-7 mutants to $P$. aeruginosa infection could also be suppressed by $h b l-1$ mutation or lin- 41 mutation [37]. Therefore, the miRNA let-7 may regulate both the MWCNTs toxicity and the innate immunity by suppressing the expressions and functions of its direct targets of HBL- 1 and LIN-41.

In C. elegans, TIR-1 and SYM-1 were identified as downstream targets for HBL-1 in the regulation of MWCNTs toxicity. On the one hand, under both normal and the MWCNTs exposure conditions, HBL-1 affects the activities of TIR-1 and SYM-1 (Fig. 5a) [33]. On the other hand, both tir-1 mutant and sym-1 mutant exhibited the similar susceptibility to MWCNTs toxicity to hbl-1 mutant (Fig. 5b and c). Mutation of tir-1 or sym-1 also caused the enhanced susceptibility to pathogen infection [39, 40]. More importantly, we observed that mutation of tir-1 or sym-1 could suppress the resistance of transgenic strain overexpressing HBL-1 to MWCNTs toxicity (Fig. 6). Therefore, HBL-1 may regulate the MWCNTs toxicity by positively affecting the TIR-1 or SYM-1 activity. That is, a signaling cascade of HBL-1TIR-1/SYM-1 was raised to be required for the regulation of MWCNTs toxicity (Fig. 9).

The gene-silencing phenomenon of RNAi is triggered by double-stranded (ds) RNA, and guides the mRNA destruction [41]. In C. elegans, alg-1 and alg-2 are two genes related to the RNAi pathway [34]. In this study, we found that alg-1 or alg-2 mutation induced a resistance to MWCNTs toxicity in inducing intestinal

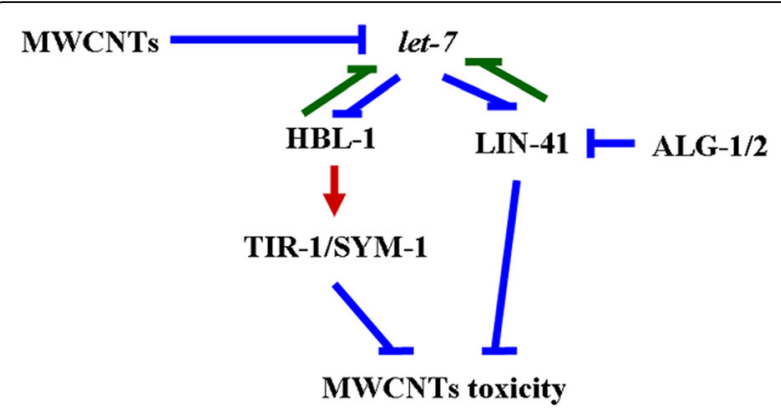

Fig. 9 A diagram showing the molecular basis for let-7 and its direst targets of HBL-1 and LIN-41 in the regulation of MWCNTS toxicity in nematodes

ROS production and in decreasing locomotion behavior (Fig. 7), implying the further involvement of molecular basis for RNAi in the regulation of MWCNTs toxicity. Moreover, we observed that lin-41 mutation suppressed the resistance of alg-1 or alg-2 mutant to MWCNTs toxicity (Fig. 7), indicating that LIN-41 may also regulate the MWCNTs toxicity by acting as the downstream target of ALG-1 or ALG-2 (Fig. 9). The genetic assay has further indicated the genetic suppression of alg-1/alg-2(RNAi) by lin-41 in regulating the heterochronic phenotypes [34].

In this study, we also observed that both HBL-1 and LIN-41, the let-7s direct targets, are responsible for the inhibition of transcription of let-7. Moreover, we found that mutation of $h b l-1$ or lin- 41 significantly increased the expression of let-7::GFP in MWCNTs exposed nematodes (Fig. 8), suggesting a feedback loop may be formed between let-7 and its targets of HBL-1 and LIN41 in the regulation of MWCNTs toxicity. That is, in MWCNTs exposed nematodes, the suppressed let-7 could be further inhibited by its targets of HBL-1 and LIN-41 (Fig. 9). Such a feedback mechanism will further strengthen the function of let-7 suppression in protecting the animals from the MWCNTs toxicity in animals.

\section{Conclusions}

In conclusion, we here investigated the potential effect of MWCNTs exposure on the molecular basis for developmental timing using in vivo assay system of C. elegans. We found that MWCNTs exposure could dysregulate the molecular basis for developmental timing as indicated by the altered expression patterns of let-7 and its targets in MWCNTs exposed nematodes. Meanwhile, we observed the resistance of let-7 mutant and the susceptibility of hbl-1 or lin-41 mutant to MWCNTs toxicity, suggesting the potential protection function of let-7 suppression in being against the MWCNTs toxicity. We further found the formation of a feedback loop between let-7 and its targets of HBL-1 and LIN-41 in the regulation of 
MWCNTs toxicity, which will further strengthen the function of let-7 suppression in protecting the nematodes from the MWCNTs toxicity. Moreover, we identified the signaling cascades of HBL-1-TIR-1/SYM-1 and ALG-1/2-LIN-41 in the regulation of MWCNTs toxicity. Our results highlight the important link between the molecular basis for developmental timing and the regulation of MWCNTs toxicity in organisms.

\section{Methods}

\section{Characterization of MWCNTs}

MWCNTs (diameter: 10-20 nm, length: 5-15 $\mu \mathrm{m}$ ) were from Shenzhen Nanotech. Port Co. Ltd. (Shenzhen, China). MWCNTs morphology in K-medium was examined by transmission electron microscopy (TEM, JEM200CX, JEOL, Japan) (Additional file 1: Figure S2A). Length distribution of MWCNTs was shown in Additional file 1: Figure S2B based on the TEM assay. The presence of $\mathrm{Ni}(0.077 \%)$ and $\mathrm{Fe}(0.017 \%)$ impurities was determined by elemental inductively coupled plasma mass spectrometer (ICPMS). After prolonged exposure, it was found that both $0.077 \% \mathrm{Ni}$ and $0.017 \% \mathrm{Fe}$ in $1000 \mu \mathrm{g} / \mathrm{L}$ MWCNTs could not induce the toxicity, such as the induction of intestinal ROS production, on nematodes [21]. Zeta potential of MWCNTs was analyzed by Nano Zetasizer (Nano ZS90, Malvern Instrument, UK). Zeta potential of MWCNTs $(1 \mathrm{mg} / \mathrm{L})$ in K-medium was $-32.9 \pm 2.9 \mathrm{mV}$.

\section{C. elegans Strains and culture}

Nematodes used in this study were wild-type N2, mutants of MT7626/let-7(mg279), CT11/hbl-1(mg285), CT8/lin-41(ma104), RF54/alg-1(gk214), RB574/alg2(ok304), RB1085/tir-1(ok1052), ZD101/tir-1(qd4), SP2163/sym-1(mn601), hbl-1(mg285)let-7(mg279), lin41(ma104); alg-1(gk214), lin-41(ma104); alg-2(ok304), and lin-41(ma104);let-7(mg279), and transgenic strains of CT12/zaEx5[let-7::GFP], lin-41(ma104);zaEx5, Ex(Phbl-1-hbl-1), tir-1(ok1052);Ex(Phbl-1-hbl-1), sym1(mn601)Ex(Phbl-1-hbl-1), and hbl-1(mg285)zaEx5. Some of these strains were from Caenorhabditis Genetics Center (funded by NIH Office of Research Infrastructure Programs (P40 OD010440)). Nematodes were maintained on nematode growth medium (NGM) plates seeded with Escherichia coli OP50 at $20{ }^{\circ} \mathrm{C}$ [9], and lysed with a bleaching mixture $(0.45 \mathrm{M} \mathrm{NaOH}, 2 \%$ $\mathrm{HOCl})$ after washing off the plates into the centrifuge tubes. The age synchronous L1-larvae population was prepared as described previously [42].

\section{Exposure and toxicity assessment}

MWCNTs were dispersed in $\mathrm{K}$ medium to prepare a stock solution $(1 \mathrm{mg} / \mathrm{mL})$. The stock MWCNTs solution was further sonicated for $30 \mathrm{~min}(40 \mathrm{kHz}, 100 \mathrm{~W})$, and diluted to the working concentrations $(10 \mu \mathrm{g} / \mathrm{L})$ with $\mathrm{K}$ medium just prior to exposure. Prolonged exposure to MWCNTs was performed from L1-larvae to young adults in 12-well sterile tissue culture plates at $20{ }^{\circ} \mathrm{C}$ in the presence of food (OP50). After MWCNTs exposure, the examined nematodes were used for the toxicity assessment using endpoints of intestinal ROS production and locomotion behavior.

The endpoint of intestinal ROS production was used to reflect the functional state of intestinal cells [43]. Intestinal ROS production was analyzed as described previously [44, 45]. After exposure, the examined nematodes were transferred to $1 \mu \mathrm{M} 5^{\prime}, 6^{\prime}$ chloromethyl-2',7'-dichlorodihydro-fluorescein diacetate $\left(\mathrm{CM}-\mathrm{H}_{2} \mathrm{DCFDA}\right.$; Molecular Probes) solution to incubate for $3 \mathrm{~h}$ in the dark. After labeling, the nematodes were mounted on a $2 \%$ agar pad for the observation and examination at $488 \mathrm{~nm}$ of excitation wavelength and $510 \mathrm{~nm}$ of emission filter under a laser scanning confocal microscope (Leica, TCS SP2, Bensheim, Germany). Relative fluorescence intensity of ROS signals in the intestine was semi-quantified and expressed as the relative fluorescence units (RFU). Sixty nematodes were examined per treatment.

The endpoint of locomotion behavior was used to reflect the functional state of motor neurons [46]. Head thrash and body bend were selected to evaluate the locomotion behavior. The head thrash and the body bend were analyzed under a dissecting microscope by eyes as described previously $[47,48]$. In C. elegans, a head thrash is defined as a change in the direction of bending at the mid body, and a body bend is defined as a change in the direction of the part of the nematodes corresponding to the posterior bulb of the pharynx along the $y$ axis, assuming that nematode was traveling along the $x$ axis. Sixty nematodes were examined per treatment.

\section{Reverse-transcription and quantitative real-time polymerase chain reaction (qRT-PCR) assay}

Total RNAs of nematodes were extracted using an RNeasy Mini kit (Qiagen), and reverse transcribed using a PrimeScript ${ }^{\mathrm{m}}$ RT reagent kit (Takara, Otsu, Shiga, Japan). After the cDNA synthesis, real-time PCR was performed using the SYBR Premix Ex Taq (Takara) for the amplification of certain PCR products. Real-time PCR was run at an optimized annealing temperature of $58{ }^{\circ} \mathrm{C}$. The relative quantification of targeted genes in comparison to a reference $t b a-1$ gene, encoding a tubulin, was determined. The final results were expressed as relative expression ratio between the targeted genes and the reference gene. All the reactions were performed for three biological replicates. For each 
biological replicate, three technical replicates were performed further. The related primer information for qRT-PCR is shown in Additional file 1: Table S1.

\section{RNAi assay}

RNAi was performed by feeding nematodes with $E$. coli strain HT115 (DE3) expressing double-stranded RNA that is homologous to a target gene as described [49]. E. coli HT115 (DE3) was prepared onto NGM containing ampicillin $(100 \mu \mathrm{g} / \mathrm{mL})$ and isopropyl 1-thio- $\beta$-D-galactopyranoside (IPTG, $5 \mathrm{mM}$ ). L1 larvae were transferred onto RNAi plates for 2 days at $20{ }^{\circ} \mathrm{C}$ until they became gravid. Gravid adults were transferred onto the fresh RNAi-expressing bacterial lawns to lay eggs so as to obtain the second generation of RNAi population.

\section{DNA constructs and transformation}

Promoter region for $h b l-1$ amplified by PCR from wildtype C. elegans genomic DNA was inserted into the pPD95_77 vector in the sense orientation. hbl-1/ F13D11.2b cDNA was amplified by PCR, and inserted into corresponding entry vector carrying the hbl-1 promoter sequence. Germline transformation was performed as described by coinjecting a testing DNA $(30 \mu \mathrm{g} / \mathrm{mL})$ and a marker DNA (Pdop-1::rfp, $60 \mu \mathrm{g} / \mathrm{mL}$ ) into the gonad of nematodes [50]. The related primer information for DNA constructions were shown in Additional file 1: Table S2.

\section{Statistical analysis}

Data in this article were expressed as means \pm standard deviation (SD). Statistical analysis was performed using SPSS 12.0 software (SPSS Inc., Chicago, USA). Differences between groups were determined using analysis of variance (ANOVA), and probability levels of 0.05 and 0.01 were considered statistically significant. Graphs were generated using Microsoft Excel software (Microsoft Corp., Redmond, WA).

\section{Additional file}

Additional file 1: Figure S1. Expression of $h b l-1$ and lin-4l in nematodes. A) Effect of let-7 mutation on expression of $h b l-1$ and lin-41 after MWCNTs exposure. Bars represent means \pm SD. ${ }^{* *} P<0.01$ vs wild-type. B) Effect of MWCNTs exposure on expression of $h b l-1$ and lin-41. Bars represent means $\pm S D .{ }^{* *} P<0.01$ vs control. Prolonged exposure was performed from L1-larvae to young adults. Exposure concentration of MWCNTs was $10 \mu \mathrm{g} / \mathrm{L}$. Figure S2. Physiochemical properties of MWCNTs. A) TEM of MWCNTs after sonication. B) Length distribution of MWCNTs after sonication. Table S1. Primer information for qRT-PCR. Table S2. Primer information for DNA constructions. (DOC $204 \mathrm{~kb})$

\section{Acknowledgements}

Not applicable.

Funding

Not applicable.
Availability of data and materials

All the data and materials are available.

\section{Authors' contributions}

DW conceived and designed the experiments. LZ and HW performed the experiments. LZ and HW analyzed the data. DW wrote the paper. All authors read and approved the final manuscript.

Ethics approval and consent to participate

Not applicable.

\section{Consent for publication}

All authors agree for the publication.

\section{Competing interests}

The authors declare that they have no competing interests.

\section{Publisher's Note}

Springer Nature remains neutral with regard to jurisdictional claims in published maps and institutional affiliations.

\section{Author details}

${ }^{1}$ Key Laboratory of Environmental Medicine Engineering in Ministry of Education, Medical School, Southeast University, Nanjing 210009, China.

${ }^{2}$ School of Public Health, Nanjing Medical University, Nanjing 211166, China.

Received: 27 March 2017 Accepted: 14 July 2017

Published online: 20 July 2017

References

1. Chen P, Lin J, Tan KL. Carbon nanotubes: a future material of life. IUBMB Life. 2000;49:105-8.

2. Gong H, Peng R, Liu Z. Carbon nanotubes for biomedical imaging: the recent advances. Adv Drug Deliv Rev. 2013;65:1951-63.

3. Gupta VK, Kumar R, Nayak A, Saleh TA, Barakat MA. Adsorptive removal of dyes from aqueous solution onto carbon nanotubes: a review. Adv Colloid Interf Sci. 2013;193-194:24-34.

4. Peng X, Chen J, Misewich JA, Wong SS. Carbon nanotube-nanocrystal heterostructures. Chem Soc Rev. 2009;38:1076-98.

5. Helland A, Wick P, Koehler A, Schmid K, Som C. Reviewing the environmental and human health knowledge base of carbon nanotubes. Environ Health Perspect. 2007;115:1125-31.

6. Simate GS, lyuke SE, Ndlovu S, Heydenrych M, Walubita LF. Human health effects of residual carbon nanotubes and traditional water treatment chemicals in drinking water. Environ Int. 2012;39:38-49.

7. Donaldson K, Aitken R, Tran L, Stone V, Duffin R, Forrest G, Alexanders A. Carbon nanotubes: a review of their properties in relation to pulmonary toxicology and workplace safety. Toxicol Sci. 2006;92:5-22.

8. Liu Y, Zhao Y, Sun B, Chen C. Understanding the toxicity of carbon nanotubes. Acc Chem Res. 2013;46:702-13.

9. Brenner S. The genetics of Caenorhabditis elegans. Genetics. 1974;77:71-94.

10. Leung MC, Williams PL, Benedetto A, Au C, Helmcke KJ, Aschner M, Meyer JN. Caenorhabditis elegans: an emerging model in biomedical and environmental toxicology. Toxicol Sci. 2008;106:5-28.

11. Yu X-M, Guan X-M, Wu Q-L, Zhao Y-L, Wang D-Y. Vitamin E ameliorates the neurodegeneration related phenotypes caused by neurotoxicity of $\mathrm{Al}_{2} \mathrm{O}_{3}-$ nanoparticles in C. elegans. Toxicol Res. 2015;4:1269-81.

12. Wang D-Y. Biological effects, translocation, and metabolism of quantum dots in nematode Caenorhabditis elegans. Toxicol Res. 2016;5:1003-11.

13. Chen P, Hsiao K, Chou C. Molecular characterization of toxicity mechanism of single-walled carbon nanotubes. Biomaterials. 2013;34:5661-9.

14. Jung SK, Qu X, Aleman-Meza B, Wang T, Riepe C, Liu Z, Li Q, Zhong W. Multi-endpoint, high-throughput study of nanomaterial toxicity in Caenorhabditis elegans. Environ Sci Technol. 2015;49:2477-85.

15. Wu Q-L, Zhou X-F, Han X-X, Zhuo Y-Z, Zhu S-T, Zhao Y-L, Wang D-Y. Genome-wide identification and functional analysis of long noncoding RNAs involved in the response to graphene oxide. Biomaterials. 2016;102: 277-91.

16. Yang J-N, Zhao Y-L, Wang Y-W, Wang H-F, Wang D-Y. Toxicity evaluation and translocation of carboxyl functionalized graphene in Caenorhabditis elegans. Toxicol Res. 2015;4:1498-510. 
17. Zhao Y-L, Wu Q-L, Li Y-P, Wang D-Y. Translocation, transfer, and in vivo safety evaluation of engineered nanomaterials in the non-mammalian alternative toxicity assay model of nematode Caenorhabditis elegans. RSC Adv. 2013;3:5741-57.

18. Nouara A, Wu Q-L, Li Y-X, Tang M, Wang H-F, Zhao Y-L, Wang D-Y. Carboxylic acid functionalization prevents the translocation of multi-walled carbon nanotubes at predicted environmental relevant concentrations into targeted organs of nematode Caenorhabditis elegans. Nano. 2013;5:6088-96.

19. Shakoor S, Sun L-M, Wang D-Y. Multi-walled carbon nanotubes enhanced fungal colonization and suppressed innate immune response to fungal infection in nematodes. Toxicol Res. 2016:5:492-9.

20. Shu C-J, Yu X-M, Wu Q-L, Zhuang Z-H, Zhang W-M, Wang D-Y. Pretreatment with paeonol prevents the adverse effects and alters the translocation of multi-walled carbon nanotubes in nematode Caenorhabditis elegans. RSC Adv. 2015:5:8942-51.

21. Wu Q-L, Li Y-X, Li Y-P, Zhao Y-L, Ge L, Wang H-F, Wang D-Y. Crucial role of biological barrier at the primary targeted organs in controlling translocation and toxicity of multi-walled carbon nanotubes in nematode Caenorhabditis elegans. Nano. 2013;5:11166-78.

22. Zhi L-T, Fu W, Wang X, Wang D-Y. ACS-22, a protein homologous to mammalian fatty acid transport protein 4 , is essential for the control of toxicity and translocation of multi-walled carbon nanotubes in Caenorhabditis elegans. RSC Adv. 2016;6:4151-9.

23. Eom HJ, Roca CP, Roh JY, Chatterjee N, Jeong JS, Shim I, Kim HM, Kim PJ, Choi K, Giralt F, Choi J. A systems toxicology approach on the mechanism of uptake and toxicity of MWCNT in Caenorhabditis elegans. Chem Biol Interact. 2015;239:153-63.

24. Bartel DP. MicroRNAs: genomics, biogenesis, mechanism, and function. Cell. 2004;116:281-97.

25. Yang R-L, Ren M-X, Rui Q, Wang D-Y. A mir-231-regulated protection mechanism against the toxicity of graphene oxide in nematode Caenorhabditis elegans. Sci Rep. 2016;6:32214.

26. Zhao Y-L, Wu Q-L, Li Y-P, Nouara A, Jia R-H, Wang D-Y. In vivo translocation and toxicity of multi-walled carbon nanotubes are regulated by microRNAs. Nano. 2014;6:4275-84

27. Zhao $Y-L$, Wu Q-L, Wang D-Y. A microRNAs-mRNAs network involved in the control of graphene oxide toxicity in Caenorhabditis elegans. RSC Adv. 2015; 5:92394-405.

28. Zhao Y-L, Yang J-N, Wang D-Y. A microRNA-mediated insulin signaling pathway regulates the toxicity of multi-walled carbon nanotubes in nematode Caenorhabditis elegans. Sci Rep. 2016;6:23234.

29. Zhuang Z-H, Li M, Liu H, Luo L-B, Gu W-D, Wu Q-L, Wang D-Y. Function of RSKS-1-AAK-2-DAF-16 signaling cascade in enhancing toxicity of multiwalled carbon nanotubes can be suppressed by mir-259 activation in Caenorhabditis elegans. Sci Rep. 2016;6:32409.

30. Sokol NS. Small temporal RNAs in animal development. Curr Opin Genet Dev. 2012;22:368-73.

31. Reinhart B, Slack F, Basson M, Pasquinelli AE, Bettinger JC, Rougvie AE, Horvitz HR, Ruvkun G. The 21 nucleotide let-7 RNA regulates $C$. elegans developmental timing. Nature. 2000;403:901-6.

32. Martinez NJ, Ow MC, Reece-Hoyes JS, Barrasa MI, Ambros VR, Walhout AJ. Genome-scale spatiotemporal analysis of Caenorhabditis elegans microRNA promoter activity. Genome Res. 2008;18:2005-15.

33. Niwa R, Hada K, Moliyama K, Ohniwa RL, Tan YM, Olsson-Carter K, Chi W, Reinke V, Slack FJ. C. elegans sym-1 is a downstream target of the hunchback-like-1 developmental timing transcription factor. Cell Cycle. 2009;8:4147-54

34. Grishok A, Pasquinelli AE, Conte D, Li N, Parrish S, Ha I, Baillie DL, Fire A, Ruvkun G, Mello CC. Genes and mechanisms related to RNA interference regulate expression of the small temporal RNAs that control C. Elegans developmental timing. Cell. 2001;106:23-34

35. Roush SF, Slack FJ. Transcription of the C. elegans let-7 microRNA is temporally regulated by one of its targets, hbl-1. Dev Biol. 2009;334:523-34.

36. Zhi L-T, Yu Y-L, Li X-Y, Wang D-Y, Wang D-Y. Molecular control of innate immune response to Pseudomonas aeruginosa infection by intestinal let-7 in Caenorhabditis elegans. PLoS Pathog. 2017;13:e1006152.

37. Ren Z, Ambros VR. Caenorhabditis elegans microRNAs of the let-7 family act in innate immune response circuits and confer robust developmental timing against pathogen stress. Proc Natl Acad Sci U S A. 2015;112:E2366-75.

38. Abrahante JE, Daul AL, Li M, Volk ML, Tennessen JM, Miller EA, Rougvie AE. The Caenorhabditis elegans hunchback-like gene lin-57/hbl-1 controls developmental time and is regulated by microRNAs. Dev Cell. 2003;4:625-37.
39. Couillault C, Pujol N, Reboul J, Sabatier L, Guichou JF, Kohara Y, Ewbank J. TLR-independent control of innate immunity in Caenorhabditis elegans by the TIR domain adaptor protein TIR-1, an ortholog of human SARM. Nat Immunol. 2004;5:488-94.

40. Fuhrman LE, Goel AK, Smith J, Shianna KV, Aballay A. Nucleolar proteins suppress Caenorhabditis elegans innate immunity by inhibiting p53/CEP-1. PLoS Genet. 2009:5:e1000657.

41. Cogoni C, Macino G. Post-transcriptional gene silencing across kingdoms. Curr Opin Genet. 2000;6:638-43.

42. Donkin S, Williams PL. Influence of developmental stage, salts and food presence on various end points using Caenorhabditis elegans for aquatic toxicity testing. Environ Toxicol Chem. 1995;14:2139-47.

43. Zhao Y-L, Yang R-L, Rui Q, Wang D-Y. Intestinal insulin signaling encodes two different molecular mechanisms for the shortened longevity induced by graphene oxide in Caenorhabditis elegans. Sci Rep. 2016;6:24024.

44. Liu Z-F, Zhou X-F, Wu Q-L, Zhao Y-L, Wang D-Y. Crucial role of intestinal barrier in the formation of transgenerational toxicity in quantum dots exposed nematodes Caenorhabditis elegans. RSC Adv. 2015;5:94257-66.

45. Yang R-L, Rui Q, Kong L, Zhang N, Li Y, Wang X-Y, Tao J, Tian P-Y, Ma Y, Wei J-R, Li G-J, Wang D-Y. Metallothioneins act downstream of insulin signaling to regulate toxicity of outdoor fine particulate matter $\left(\mathrm{PM}_{2.5}\right)$ during spring festival in Beijing in nematode Caenorhabditis elegans. Toxicol Res. 2016;5:1097-105

46. Chen H, Li H-R, Wang D-Y. Graphene oxide dysregulates Neuroligin/NLG-1mediated molecular signaling in interneurons in Caenorhabditis elegans. Sci Rep. 2017;7:41655.

47. Zhao Y-L, Jia R-H, Qiao Y, Wang D-Y. Glycyrrhizic acid, active component from Glycyrrhizae radix, prevents toxicity of graphene oxide by influencing functions of microRNAs in nematode Caenorhabditis elegans. Nanomedicine: Nanotechnol Biol Med. 2016;12:735-44

48. Zhi L-T, Qu M, Ren M-X, Zhao L, Li Y-H, Wang D-Y. Graphene oxide induces canonical Wnt/ $\beta$-catenin signaling-dependent toxicity in Caenorhabditis elegans. Carbon. 2017;113:122-31.

49. Wu Q-L, Cao X-O, Yan D, Wang D-Y, Aballay A. Genetic screen reveals link between maternal-effect sterile gene mes-1 and P. Aeruginosa-induced neurodegeneration in C. Elegans. J Biol Chem. 2015;290:29231-9.

50. Mello C, Fire A. DNA transformation. Methods Cell Biol. 1995:48:451-82.

\section{Submit your next manuscript to BioMed Central and we will help you at every step:}

- We accept pre-submission inquiries

- Our selector tool helps you to find the most relevant journal

- We provide round the clock customer support

- Convenient online submission

- Thorough peer review

- Inclusion in PubMed and all major indexing services

- Maximum visibility for your research

Submit your manuscript at www.biomedcentral.com/submit

) Biomed Central 